\title{
$q$-Counting descent pairs with prescribed tops and bottoms
}

\author{
John Hall ${ }^{\dagger} \quad$ Jeffrey Liese ${ }^{\ddagger} \quad$ Jeffrey B. Remmel ${ }^{\star}$
}

Submitted: Oct 12, 2008; Accepted: Aug 25, 2009; Published: Aug 31, 2009

Mathematics Subject Classification: 05A05, 05A15

\begin{abstract}
Given sets $X$ and $Y$ of positive integers and a permutation $\sigma=\sigma_{1} \sigma_{2} \cdots \sigma_{n} \in S_{n}$, an $(X, Y)$-descent of $\sigma$ is a descent pair $\sigma_{i}>\sigma_{i+1}$ whose "top" $\sigma_{i}$ is in $X$ and whose "bottom" $\sigma_{i+1}$ is in $Y$. Recently Hall and Remmel [4] proved two formulas for the number $P_{n, s}^{X, Y}$ of $\sigma \in S_{n}$ with $s(X, Y)$-descents, which generalized Liese's results in [1]. We define a new statistic stat $\operatorname{st}_{X, Y}(\sigma)$ on permutations $\sigma$ and define $P_{n, s}^{X, Y}(q)$ to be the sum of $q^{\text {stat }_{X, Y}(\sigma)}$ over all $\sigma \in S_{n}$ with $s(X, Y)$-descents. We then show that there are natural $q$-analogues of the Hall-Remmel formulas for $P_{n, s}^{X, Y}(q)$.
\end{abstract}

\section{Introduction}

Let $S_{n}$ denote the set of permutations of the set $[n]=\{1,2, \ldots, n\}$. Given subsets $X, Y \subseteq \mathbb{N}$ and a permutation $\sigma \in S_{n}$, let

$$
\begin{aligned}
\operatorname{Des}_{X, Y}(\sigma) & =\left\{i: \sigma_{i}>\sigma_{i+1} \& \sigma_{i} \in X \& \sigma_{i+1} \in Y\right\}, \text { and } \\
\operatorname{des}_{X, Y}(\sigma) & =\left|\operatorname{Des}_{X, Y}(\sigma)\right| .
\end{aligned}
$$

If $i \in \operatorname{Des}_{X, Y}(\sigma)$, then we call the pair $\left(\sigma_{i}, \sigma_{i+1}\right)$ an $(X, Y)$-descent. For example, if $X=\{2,3,5\}, Y=\{1,3,4\}$, and $\sigma=54213$, then $\operatorname{Des}_{X, Y}(\sigma)=\{1,3\}$ and $\operatorname{des}_{X, Y}(\sigma)=2$.

For fixed $n$ we define the polynomial

$$
P_{n}^{X, Y}(x)=\sum_{s \geqslant 0} P_{n, s}^{X, Y} x^{s}:=\sum_{\sigma \in S_{n}} x^{\operatorname{des}_{X, Y}(\sigma)} .
$$

\footnotetext{
${ }^{\dagger}$ Department of Mathematics, Harvard University, Cambridge, MA, hall@math.harvard.edu

${ }_{\ddagger}^{\ddagger}$ Department of Mathematics, California Polytechnic State University, San Luis Obispo, CA 93407, jliese@calpoly.edu

*Department of Mathematics, University of California, San Diego, La Jolla, CA 92093, jremmel@ucsd.edu

*This work partially supported by NSF grant DMS 0654060
} 
Thus the coefficient $P_{n, s}^{X, Y}$ is the number of $\sigma \in S_{n}$ with exactly $s(X, Y)$-descents.

Hall and Remmel [4] gave direct combinatorial proofs of a pair of formulas for $P_{n, s}^{X, Y}$. First of all, for any set $A \subseteq \mathbb{N}$, let

$$
\begin{aligned}
& A_{n}=A \cap[n], \text { and } \\
& A_{n}^{c}=\left(A^{c}\right)_{n}=[n]-A .
\end{aligned}
$$

Then Hall and Remmel [4] proved the following theorem.

\section{Theorem 1.1.}

$$
P_{n, s}^{X, Y}=\left|X_{n}^{c}\right| ! \sum_{r=0}^{s}(-1)^{s-r}\left(\begin{array}{c}
\left|X_{n}^{c}\right|+r \\
r
\end{array}\right)\left(\begin{array}{c}
n+1 \\
s-r
\end{array}\right) \prod_{x \in X_{n}}\left(1+r+\alpha_{X, n, x}+\beta_{Y, n, x}\right),
$$

and

$$
P_{n, s}^{X, Y}=\left|X_{n}^{c}\right| ! \sum_{r=0}^{\left|X_{n}\right|-s}(-1)^{\left|X_{n}\right|-s-r}\left(\begin{array}{c}
\left|X_{n}^{c}\right|+r \\
r
\end{array}\right)\left(\begin{array}{c}
n+1 \\
\left|X_{n}\right|-s-r
\end{array}\right) \prod_{x \in X_{n}}\left(r+\beta_{X, n, x}-\beta_{Y, n, x}\right),
$$

where for any set $A$ and any $j, 1 \leqslant j \leqslant n$, we define

$$
\begin{aligned}
& \alpha_{A, n, j}=\left|A^{c} \cap\{j+1, j+2, \ldots, n\}\right|=|\{x: j<x \leqslant n \& x \notin A\}|, \text { and } \\
& \beta_{A, n, j}=\left|A^{c} \cap\{1,2, \ldots, j-1\}\right|=|\{x: 1 \leqslant x<j \& x \notin A\}| .
\end{aligned}
$$

Example 1.2. Suppose $X=\{2,3,4,6,7,9\}, Y=\{1,4,8\}$, and $n=6$. Thus $X_{6}=$ $\{2,3,4,6\}, X_{6}^{c}=\{1,5\}, Y_{6}=\{1,4\}, Y_{6}^{c}=\{2,3,5,6\}$, and we have the following table of values of $\alpha_{X, 6, x}, \beta_{Y, 6, x}$, and $\beta_{X, 6, x}$.

\begin{tabular}{|c|c|c|c|c|}
\hline$x$ & 2 & 3 & 4 & 6 \\
\hline$\alpha_{X, 6, x}$ & 1 & 1 & 1 & 0 \\
\hline$\beta_{Y, 6, x}$ & 0 & 1 & 2 & 3 \\
\hline$\beta_{X, 6, x}$ & 1 & 1 & 1 & 2 \\
\hline
\end{tabular}

Equation (1.2) gives

$$
\begin{aligned}
P_{6,2}^{X, Y} & =2 ! \sum_{r=0}^{2}(-1)^{2-r}\left(\begin{array}{c}
2+r \\
r
\end{array}\right)\left(\begin{array}{c}
7 \\
2-r
\end{array}\right)(2+r)(3+r)(4+r)(4+r) \\
& =2(1 \cdot 21 \cdot 2 \cdot 3 \cdot 4 \cdot 4-3 \cdot 7 \cdot 3 \cdot 4 \cdot 5 \cdot 5+6 \cdot 1 \cdot 4 \cdot 5 \cdot 6 \cdot 6) \\
& =2(2016-6300+4320) \\
& =72 .
\end{aligned}
$$

while (1.3) gives

$$
\begin{aligned}
P_{6,2}^{X, Y} & =2 ! \sum_{r=0}^{2}(-1)^{2-r}\left(\begin{array}{c}
2+r \\
r
\end{array}\right)\left(\begin{array}{c}
7 \\
2-r
\end{array}\right)(1+r)(0+r)(-1+r)(-1+r) \\
& =2(1 \cdot 21 \cdot 1 \cdot 0 \cdot(-1) \cdot(-1)-3 \cdot 7 \cdot 2 \cdot 1 \cdot 0 \cdot 0+6 \cdot 1 \cdot 3 \cdot 2 \cdot 1 \cdot 1) \\
& =2(0-0+36) \\
& =72 .
\end{aligned}
$$


The main goal of this paper is to prove $q$-analogues of (1.2) and (1.3). Let

$$
\begin{aligned}
{[n]_{q} } & =1+q+q^{2}+\ldots+q^{n-1}, \\
{[n]_{q} ! } & =[n]_{q}[n-1]_{q} \cdots[2]_{q}[1]_{q}, \\
{\left[\begin{array}{c}
n \\
k
\end{array}\right]_{q} } & =\frac{[n]_{q} !}{[k]_{q} ![n-k]_{q} !}, \\
{[a]_{n} } & =[a]_{q}[a+1]_{q} \cdots[a+n-1]_{q}, \\
(a)_{\infty} & =(a ; q)_{\infty}=\prod_{k=0}^{\infty}\left(1-a q^{k}\right), \text { and } \\
(a)_{n} & =(a ; q)_{n}=\frac{(a)_{\infty}}{\left(a q^{n}\right)_{\infty} .}
\end{aligned}
$$

There are two natural approaches to finding $q$-analogues of (1.2) and (1.3). The first approach is to use $q$-analogues of the simple recursions that are satisfied by the coefficients $P_{n, s}^{X, Y}$. This approach naturally leads us to recursively define of a pair of statistics $\operatorname{stat}_{X, Y}(\sigma)$ and $\overline{\operatorname{stat}}_{X, Y}(\sigma)$ on permutations $\sigma$ so that if we define

$$
P_{n, s}^{X, Y}(q)=\sum_{\sigma \in S_{n}, \operatorname{des}_{X, Y}(\sigma)=s} q^{\operatorname{stat}_{X, Y}(\sigma)}
$$

and

$$
\bar{P}_{n, s}^{X, Y}(q)=\sum_{\sigma \in S_{n}, \operatorname{des}_{X, Y}(\sigma)=s} q^{\overline{\operatorname{stat}}_{X, Y}(\sigma)},
$$

then we can prove the following formulas:

$$
\begin{aligned}
P_{n, s}^{X, Y}(q)= & {\left[\left|X_{n}^{c}\right|\right]_{q} ! \sum_{r=0}^{s}(-1)^{s-r}\left(q^{\left(\frac{s-r}{2}\right)}\left[\begin{array}{c}
\left|X_{n}^{c}\right|+r \\
r
\end{array}\right]_{q}\left[\begin{array}{c}
n+1 \\
s-r
\end{array}\right]_{q} .\right.} \\
& \left.\prod_{x \in X_{n}}\left[1+r+\alpha_{X, n, x}+\beta_{Y, n, x}\right]_{q}\right)
\end{aligned}
$$

and

$$
\begin{aligned}
\bar{P}_{n, s}^{X, Y}(q)= & {\left[\left|X_{n}^{c}\right|\right]_{q} ! \sum_{r=0}^{\left|X_{n}\right|-s}\left((-1)^{\left|X_{n}\right|-s-r} q^{\left(\left|X_{n}\right|-s-r\right.}\right)\left[\begin{array}{c}
\left|X_{n}^{c}\right|+r \\
r
\end{array}\right]_{q}\left[\begin{array}{c}
n+1 \\
s-r
\end{array}\right]_{q} . } \\
& \left.\prod_{x \in X_{n}}\left[r+\beta_{X, n, x}-\beta_{Y, n, x}\right]_{q}\right) .
\end{aligned}
$$

The second approach is to $q$-analogue the combinatorial proofs of (1.2) and (1.3). We will see that this approach also works and leads to a more direct definition of $\operatorname{stat}_{X, Y}(\sigma)$ and $\overline{\operatorname{stat}}_{X, Y}(\sigma)$, involving generalizations of classical permutation statistics such as inv and maj. 
The outline of this paper is as follows. In Section 2, we shall give $q$-analogues of the basic recursions developed by Hall and Remmel [4] for the coefficients $P_{n, s}^{X, Y}$ and give the recursive definitions of $\operatorname{stat}_{X, Y}(\sigma)$ and $\overline{\operatorname{stat}}_{X, Y}(\sigma)$. In Section 3, we shall give a direct combinatorial proof of (1.6) and (1.7). In Section 4, we shall use some basic hypergeometric series identities to show that in certain special cases, the formulas (1.6) and (1.7) can be significantly simplified. For example, we shall show that when $Y$ is the set of natural numbers $\mathbb{N}$ and $X$ is the set of even numbers $2 \mathbb{N}$, then

$$
P_{2 n, s}^{X, Y}(q)=q^{s^{2}}\left([n]_{q} !\right)^{2}\left[\begin{array}{c}
n \\
s
\end{array}\right]_{q}^{2}
$$

which was first proved by Liese and Remmel [5] by recursion. We will also describe the equality of (1.6) and (1.7) as a special case of a $q$-analogue of a transformation of Karlsson-Minton type hypergeometric series due to Gasper [2].

\section{Recursions for $P_{n, s}^{X, Y}(q)$}

In this section, we shall give $q$-analogues of the recursions for the coefficients $P_{n, s}^{X, Y}$ developed by Hall and Remmel [4].

Given $X, Y \subseteq \mathbb{N}$, let $P_{0}^{X, Y}(x, y)=1$, and for $n \geqslant 1$, define

$$
P_{n}^{X, Y}(x, y)=\sum_{s, t \geqslant 0} P_{n, s, t}^{X, Y} x^{s} y^{t}:=\sum_{\sigma \in S_{n}} x^{\operatorname{des}_{X, Y}(\sigma)} y^{\left|Y_{n}^{c}\right|} .
$$

Let $\Phi_{n+1}$ and $\Psi_{n+1}$ be the operators defined as

$$
\begin{aligned}
& \Phi_{n+1}: x^{s} y^{t} \longrightarrow s x^{s-1} y^{t}+(n+1-s) x^{s} y^{t} \\
& \Psi_{n+1}: x^{s} y^{t} \longrightarrow(s+t+1) x^{s} y^{t}+(n-s-t) x^{s+1} y^{t} .
\end{aligned}
$$

Then Hall and Remmel proved the following.

Proposition 2.1. For any sets $X, Y \subseteq \mathbb{N}$, the polynomials $P_{n}^{X, Y}(x, y)$ satisfy

$$
P_{n+1}^{X, Y}(x, y)=\left\{\begin{array}{lll}
y \cdot & \Phi_{n+1}\left(P_{n}^{X, Y}(x, y)\right) & \text { if } n+1 \notin X \text { and } n+1 \notin Y, \\
& \Phi_{n+1}\left(P_{n}^{X, Y}(x, y)\right) & \text { if } n+1 \notin X \text { and } n+1 \in Y, \\
y \cdot & \Psi_{n+1}\left(P_{n}^{X, Y}(x, y)\right) & \text { if } n+1 \in X \text { and } n+1 \notin Y, \\
& \Psi_{n+1}\left(P_{n}^{X, Y}(x, y)\right) & \text { if } n+1 \in X \text { and } n+1 \in Y .
\end{array}\right.
$$

It is easy to see that Proposition 2.1 implies that the following recursion holds for the coefficients $P_{n, s, t}^{X, Y}$ for all $X, Y \subseteq \mathbb{N}$ and $n \geqslant 1$.

$$
\begin{aligned}
& P_{n+1, s, t}^{X, Y}= \\
& \begin{cases}(s+1) P_{n, S+1, t-1}^{X, Y}+(n+1-s) P_{n, s, t-1}^{X, Y} & \text { if } n+1 \notin X \text { and } n+1 \notin Y, \\
(s+1) P_{n, S+1, t}^{X+(n+1)}+(n+1-s) P_{n, s, t}^{X, Y} & \text { if } n+1 \notin X \text { and } n+1 \in Y, \\
(s+t) P_{n, s, t-1}^{X, Y}+(n+2-s-t) P_{n, Y-1, t-1}^{X, 1} & \text { if } n+1 \in X \text { and } n+1 \notin Y, \text { and } \\
(s+t+1) P_{n, s, t}^{X, Y}+(n+1-s-t) P_{n, s-1, t}^{X, Y} & \text { if } n+1 \in X \text { and } n+1 \in Y .\end{cases}
\end{aligned}
$$


We define two $q$-analogues of the operators $\Phi_{n+1}$ and $\Psi_{n+1}$ as follows. Let $\Phi_{n+1}^{q}$ and $\Psi_{n+1}^{q}$ be the operators defined as

$$
\begin{array}{ll}
\Phi_{n+1}^{q}: & x^{s} y^{t} \longrightarrow[s]_{q} x^{s-1} y^{t}+q^{s}[n+1-s]_{q} x^{s} y^{t} \\
\Psi_{n+1}^{q} & : \quad x^{s} y^{t} \longrightarrow[s+t+1]_{q} x^{s} y^{t}+q^{s+t+1}[n-s-t]_{q} x^{s+1} y^{t},
\end{array}
$$

and let $\bar{\Phi}_{n+1}^{q}$ and $\bar{\Psi}_{n+1}^{q}$ be the operators defined as

$$
\begin{array}{ll}
\bar{\Phi}_{n+1}^{q}: & x^{s} y^{t} \longrightarrow q^{n+1-s}[s]_{q} x^{s-1} y^{t}+[n+1-s]_{q} x^{s} y^{t} \\
\bar{\Psi}_{n+1}^{q} & : \quad x^{s} y^{t} \longrightarrow q^{n-s-t}[s+t+1]_{q} x^{s} y^{t}+[n-s-t]_{q} x^{s+1} y^{t} .
\end{array}
$$

Given subsets $X, Y \subseteq \mathbb{N}$, we define the polynomials $P_{n}^{X, Y}(q, x, y)$ by $P_{0}^{X, Y}(q, x, y)=1$ and

$$
P_{n+1}^{X, Y}(q, x, y)=\left\{\begin{array}{lll}
y \cdot & \Phi_{n+1}^{q}\left(P_{n}^{X, Y}(q, x, y)\right), \quad \text { if } n+1 \notin X, n+1 \notin Y, \\
& \Phi_{n+1}^{q}\left(P_{n}^{X, Y}(q, x, y)\right), \quad \text { if } n+1 \notin X, n+1 \in Y, \\
y \cdot & \Psi_{n+1}^{q}\left(P_{n}^{X, Y}(q, x, y)\right), \quad \text { if } n+1 \in X, n+1 \notin Y, \text { and } \\
& \Psi_{n+1}^{q}\left(P_{n}^{X, Y}(q, x, y)\right), \quad \text { if } n+1 \in X, n+1 \in Y .
\end{array}\right.
$$

Similarly, we define the polynomials $\bar{P}_{n}^{X, Y}(q, x, y)$ by $\bar{P}_{0}^{X, Y}(q, x, y)=1$ and

$$
\bar{P}_{n+1}^{X, Y}(q, x, y)=\left\{\begin{array}{lll}
y \cdot & \bar{\Phi}_{n+1}^{q}\left(\bar{P}_{n}^{X, Y}(q, x, y)\right), & \text { if } n+1 \notin X, n+1 \notin Y, \\
& \bar{\Phi}_{n+1}^{q}\left(\bar{P}_{n}^{X, Y}(q, x, y)\right), & \text { if } n+1 \notin X, n+1 \in Y, \\
y \cdot & \bar{\Psi}_{n+1}^{q}\left(\bar{P}_{n}^{X, Y}(q, x, y)\right), & \text { if } n+1 \in X, n+1 \notin Y, \text { and } \\
& \bar{\Psi}_{n+1}^{q}\left(\bar{P}_{n}^{X, Y}(q, x, y)\right), & \text { if } n+1 \in X, n+1 \in Y .
\end{array}\right.
$$

It is easy to see that (2.2) implies that

$$
P_{n+1, s, t}^{X, Y}(q)=\left\{\begin{array}{r}
{[s+1]_{q} P_{n, s+1, t-1}^{X, Y}(q)+q^{s}[n+1-s]_{q} P_{n, s, t-1}^{X, Y}(q)} \\
\quad \text { if } n+1 \notin X \text { and } n+1 \notin Y, \\
{[s+1]_{q} P_{n, s+1, t}^{X, Y}(q)+q^{s}[n+1-s]_{q} P_{n, s, t}^{X, Y}(q)} \\
\quad \text { if } n+1 \notin X \text { and } n+1 \in Y, \\
{[s+t]_{q} P_{n, s, t-1}^{X, Y}(q)+q^{s+t-1}[n+2-s-t]_{q} P_{n, s-1, t-1}^{X, Y}(q)} \\
\text { if } n+1 \in X \text { and } n+1 \notin Y, \\
{[s+t+1]_{q} P_{n, s, t}^{X, Y}(q)+q^{s+t}[n+1-s-t]_{q} P_{n, s-1, t}^{X, Y}(q)} \\
\text { if } n+1 \in X \text { and } n+1 \in Y .
\end{array}\right.
$$

Next we describe an insertion statistic $\operatorname{stat}_{X, Y}(\sigma)$, which generalizes a statistic introduced in [5], so that

$$
P_{n}^{X, Y}(q, x, y)=\sum_{\sigma \in S_{n}} q^{\text {stat } X, Y(\sigma)} x^{\operatorname{des}_{X, Y}(\sigma)} y^{\left|Y_{n}^{c}\right|}
$$

We define $\operatorname{stat}_{X, Y}(\sigma)$ by recursion. For any $\sigma=\sigma_{1} \cdots \sigma_{n} \in S_{n}$, there are $n+1$ positions where we can insert $n+1$ to obtain a permutation in $S_{n+1}$. That is, we either insert $n+1$ at 
the end or immediately before $\sigma_{i}$ for $i=1, \ldots, n$. We next describe a labeling procedure for these possible positions. That is, if $n+1 \notin X$, then we first label positions which are between an $X, Y$-descent from left to right with the integers from 0 to $\operatorname{des}_{X, Y}(\sigma)-1$ and then label the remaining positions from right to left with the integers from $\operatorname{des}_{X, Y}(\sigma)$ to $n$. If $n+1 \in X$, then we label the positions which lie between an $X, Y$-descent or are immediately in front of an element of $Y_{n}^{c}$ plus the position at the end from left to right with the integers $0, \ldots, \operatorname{des}_{X, Y}(\sigma)+\left|Y_{n}^{c}\right|$ and then label the remaining positions from right to left with the integers $\operatorname{des}_{X, Y}(\sigma)+\left|Y_{n}^{c}\right|+1$ to $n$.

Example 2.2. Suppose that $X_{7}=\{2,3,6,7\}$ and $Y_{7}=\{1,2,3,4\}$ and $\sigma=6314572$. Then if $8 \notin X$, then we would label the positions of $\sigma$ as

$$
\sigma={ }_{\overline{7}} 6_{\overline{0}} 3_{\overline{1}} 1_{\overline{6}} 4_{\overline{5}} 5_{\overline{4}} 7_{\overline{2}} 2_{\overline{3}} .
$$

If $8 \in X$, then we would label the positions of $\sigma$ as

$$
\sigma={ }_{\overline{0}} \quad 6_{\overline{1}} 3_{\overline{2}} 1_{\overline{7}} 4_{\overline{3}} 5_{\overline{4}} 7_{\overline{5}} 2_{\overline{6}} .
$$

We then define $\sigma^{(k)}$ to be the permutation in $S_{n+1}$ that is obtained by inserting $n+1$ into the position labeled with a $k$ using the above labeling scheme and recursively define $\operatorname{stat}_{X, Y}(\sigma)$ by declaring that

1. $\operatorname{stat}_{X, Y}(\sigma)=0$ if $\sigma \in S_{1}$ and

2. $\operatorname{stat}_{X, Y}(\sigma)=\operatorname{stat}_{X, Y}(\tau)+k$ if $\sigma=\tau^{(k)}$ for some $\tau \in S_{n}$ if $\sigma \in S_{n+1}$.

Example 2.3. Suppose that $X_{7}=\{2,3,6,7\}$ and $Y_{7}=\{1,2,3,4\}$ and $\sigma=6314572$.

\begin{tabular}{|c|c|}
\hline$\sigma$ restricted to $\{1, \ldots, k\}$ & Contribution to stat $_{X, Y}(\sigma)$ \\
\hline$\sigma \Upsilon_{\{1\}}={ }_{1} 1_{\overline{0}}$ & 0 \\
\hline$\sigma \Upsilon_{\{1,2\}}={ }_{2} 1_{-1} 2_{-}$ & 0 \\
\hline 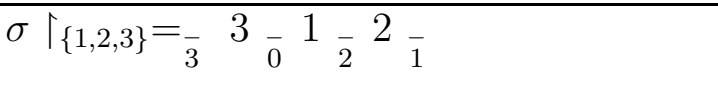 & 2 \\
\hline$\sigma \uparrow_{\{1,2,3,4\}}={ }_{\overline{4}} 3_{\overline{0}} 1_{\overline{3}} 4_{\overline{2}} 2_{\overline{1}}$ & 2 \\
\hline$\sigma \Upsilon_{\{1,2,3,4,5\}}={ }_{\overline{5}} 3_{\overline{0}} 1_{\overline{4}} 4_{\overline{1}} 5_{\overline{3}}{ }^{2} \overline{2}$ & 2 \\
\hline$\sigma\left\lceil\{1,2,3,4,5,6\}={ }_{0} 6_{\overline{1}} 3_{\overline{2}} 1_{\overline{6}} 4_{\overline{3}} 5 \overline{5}_{\overline{5}} 2_{\overline{4}}\right.$ & 5 \\
\hline$\sigma=6314572$ & 5 \\
\hline
\end{tabular}
Then we can compute stat ${ }_{X, Y}(\sigma)$ by recursion using the labeling scheme as follows. 
Thus $\operatorname{stat}_{X, Y}(\sigma)=16$ in this case.

Note that for any $\sigma \in S_{n}$,

$$
\sum_{k=0}^{n} q^{\text {stat } X, Y\left(\sigma^{(k)}\right)}=\left(1+q+\cdots+q^{n}\right) q^{\operatorname{stat}_{X, Y}(\sigma)}=[n+1]_{q} q^{\operatorname{stat}_{X, Y}(\sigma)}
$$

from which it easily follows by induction that

$$
\sum_{\sigma \in S_{n}} q^{\operatorname{stat}_{X, Y}(\sigma)}=[n]_{q} !
$$

Thus our statistic is Mahonian. Moreover, it is easy to check that if we define

$$
P_{n, s, t}^{X, Y}(q)=\sum_{\sigma \in S_{n}, \operatorname{des}_{X, Y}(\sigma)=s,\left|Y_{n}^{c}\right|=t} q^{\operatorname{stat}_{X, Y}(\sigma)}
$$

then the $P_{n, s, t}^{X, Y}(q)$ 's satisfy the recursions (2.4). For example, suppose that $n+1 \notin X$ and $n+1 \notin Y$. Then to obtain a permutation $\sigma \in S_{n+1}$ which contributes to $P_{n+1, s, t}^{X, Y}(q)$, we can either (i) start with an element $\alpha \in S_{n}$ such that $\operatorname{des}_{X, Y}(\alpha)=s+1$ and insert $n+1$ in any position that lies between an $X, Y$-descent in $\alpha$ because that will destroy that $X, Y$-descent or (ii) start with an element $\beta \in S_{n}$ such that $\operatorname{des}_{X, Y}(\beta)=s$ and insert $n+1$ in any position other than those that lie between an $X, Y$-descent in $\beta$ since such an insertion will preserve the number of $X, Y$-descents. In case (i), our labeling ensures that such an $\alpha$ will contribute $\left(1+q+\cdots+q^{s}\right) q^{\text {stat }_{X, Y}(\alpha)}=[s+1]_{q} q^{\text {stat }_{X, Y}(\alpha)}$ to $P_{n+1, s, t}^{X, Y}(q)$ so that we get a total contribution of $[s+1]_{q} P_{n, s+1, t}^{X, Y}(q)$ to $P_{n+1, s, t}^{X, Y}(q)$ from the permutations in case (i). Similarly, our labeling ensures that each such $\beta$ contributes $\left(q^{s}+\cdots+q^{n}\right) q^{\text {stat }_{X, Y}(\beta)}=q^{s}[n+1-s]_{q}$ to $P_{n+1, s, t}^{X, Y}(q)$ so that we get a total contribution of $q^{s}[n+1-s]_{q} P_{n, s+1, t}^{X, Y}(q)$ to $P_{n+1, s, t}^{X, Y}(q)$ from the permutations in case (ii). The other cases are proved in a similar manner.

It is easy to see that (2.3) implies that

$$
\bar{P}_{n+1, s, t}^{X, Y}(q)=\left\{\begin{array}{r}
q^{n-s}[s+1]_{q} \bar{P}_{n, s+1, t-1}^{X, Y}(q)+[n+1-s]_{q} \bar{P}_{n, s, t-1}^{X, Y}(q) \\
\text { if } n+1 \notin X \text { and } n+1 \notin Y, \\
q^{n-s}[s+1]_{q} \bar{P}_{n, s+1, t}^{X, Y}(q)+[n+1-s]_{q} \bar{P}_{n, s, t}^{X, Y}(q) \\
\text { if } n+1 \notin X \text { and } n+1 \in Y, \\
q^{n+1-s-t}[s+t]_{q} \bar{P}_{n, s, t-1}^{X, Y}(q)+[n+2-s-t]_{q} \bar{P}_{n, s-1, t-1}^{X, Y}(q) \\
\text { if } n+1 \in X \text { and } n+1 \notin Y, \\
q^{n-s-t}[s+t+1]_{q} \bar{P}_{n, s, t}^{X, Y}(q)+[n+1-s-t]_{q} \bar{P}_{n, s-1, t}^{X, Y}(q) \\
\text { if } n+1 \in X \text { and } n+1 \in Y .
\end{array}\right.
$$

Again we can recursively define an insertion statistic $\overline{\operatorname{stat}}_{X, Y}(\sigma)$ so that

$$
\bar{P}_{x}^{X, Y}(q, x, y)=\sum_{\sigma \in S_{n}} q^{\overline{\operatorname{stat}}_{X, Y}(\sigma)} x^{\operatorname{des}_{X, Y}(\sigma)} y^{\left|Y_{n}^{c}\right|}
$$


The only difference in this case is that if a possible insertion position $p$ was labeled with $i$ relative to stat ${ }_{X, Y}$, then position $p$ should be labeled with $n-i$ relative to $\overline{\operatorname{stat}}_{X, Y}$. It is easy to see that this labeling can be described as follows. If $n+1 \notin X$, then we first label positions which are not between an $X, Y$-descent from left to right with the integers from 0 to $n-\operatorname{des}_{X, Y}(\sigma)$ and then label the remaining positions from right to left with the integers from $n-\operatorname{des}_{X, Y}(\sigma)+1$ to $n$. If $n+1 \in X$, then we label the positions which do not lie between an $X, Y$-descent or are not immediately in front of an element of $Y_{n}^{c}$ or are not at the end from left to right with the integers $0, \ldots, n-\left(\operatorname{des}_{X, Y}(\sigma)+\left|Y_{n}^{c}\right|\right)-1$ and then label the remaining positions from right to left with the integers $n-\left(\operatorname{des}_{X, Y}(\sigma)+\left|Y_{n}^{c}\right|\right)$ to $n$.

Example 2.4. Suppose that $X_{7}=\{2,3,6,7\}$ and $Y_{7}=\{1,2,3,4\}$ and $\sigma=6314572$. Then if $8 \notin X$, then we would label the positions of $\sigma$ as

$$
\sigma={ }_{0} \quad 6_{\overline{7}} 3_{\overline{6}} 1_{\overline{1}} 4_{\overline{2}} 5_{\overline{3}} 7_{\overline{5}} 2_{-} .
$$

If $8 \in X$, then we would label the positions of $\sigma$ as

$$
\sigma={ }_{\overline{7}} 6_{\overline{6}} 3_{\overline{5}} 1_{\overline{0}} 4_{\overline{4}} 5_{\overline{3}} 7_{\overline{2}} 2_{\overline{1}} .
$$

We then define $\sigma^{(\bar{k})}$ to be the permutation in $S_{n+1}$ that is obtained by inserting $n+1$ into the position labeled with a $k$ using the above labeling scheme and recursively define $\overline{\operatorname{stat}}_{X, Y}(\sigma)$ by declaring that

1. $\overline{\operatorname{stat}}_{X, Y}(\sigma)=0$ if $\sigma \in S_{1}$ and

2. $\overline{\operatorname{stat}}_{X, Y}(\sigma)=\overline{\operatorname{stat}}_{X, Y}(\tau)+k$ if $\sigma=\tau^{(\bar{k})}$ for some $\tau \in S_{n}$ if $\sigma \in S_{n+1}$.

Example 2.5. Suppose that $X_{7}=\{2,3,6,7\}$ and $Y_{7}=\{1,2,3,4\}$ and $\sigma=6314572$.

\begin{tabular}{|c|c|}
\hline$\sigma$ restricted to $\{1, \ldots, k\}$ & Contribution to $\overline{\text { stat }}_{X, Y}(\sigma)$ \\
\hline$\sigma \Upsilon_{\{1\}}={ }_{0} 1_{\overline{1}}$ & 0 \\
\hline$\sigma \Upsilon_{\{1,2\}}={ }_{0} 1_{-1} 2_{-}$ & 1 \\
\hline$\sigma \Upsilon_{\{1,2,3\}}={ }_{0} 3_{\overline{3}} 1_{\overline{1}} 2_{\overline{2}}$ & 0 \\
\hline$\sigma \Upsilon_{\{1,2,3,4\}}=\overline{0}_{\overline{0}} 3_{\overline{4}} 1_{\overline{1}} 4_{\overline{2}} 2_{\overline{3}}$ & 1 \\
\hline$\sigma \uparrow_{\{1,2,3,4,5\}}={ }_{0} 3_{\overline{5}} 1_{\overline{1}} 4_{\overline{4}} 5_{\overline{2}} 2_{\overline{3}}$ & 2 \\
\hline$\sigma\left\lceil\{1,2,3,4,5,6\}={ }_{\overline{6}} 6_{\overline{5}} 3_{\overline{4}} 1_{\overline{0}} 4_{\overline{3}} 5_{\overline{1}} 2_{\overline{2}}\right.$ & 0 \\
\hline$\sigma=6314572$ & 1 \\
\hline
\end{tabular}
Then we can compute $\overline{\text { stat }}_{X, Y}(\sigma)$ by recursion using the labeling scheme as follows. 
Thus $\overline{\text { stat }}_{X, Y}(\sigma)=5$ in this case.

Again it is easy to check that

$$
\sum_{\sigma \in S_{n}} q^{\overline{\operatorname{stat}}_{X, Y}(\sigma)}=[n]_{q} !
$$

so that $\overline{\operatorname{stat}}_{X, Y}$ is also a Mahonian statistic. We then define

$$
\bar{P}_{n, s, t}^{X, Y}(q)=\sum_{\sigma \in S_{n}, \operatorname{des}_{X, Y}(\sigma)=s,\left|Y_{n}^{c}\right|=t} q^{\overline{\operatorname{stat}}_{X, Y}(\sigma)} .
$$

Again is straightforward to see that the $\bar{P}_{n, s, t}^{X, Y}(q)$ satisfy the recursion (2.7). Finally, it is easy to prove by induction that if $\sigma \in S_{n}$, then

$$
\operatorname{stat}_{X, Y}(\sigma)=\left(\begin{array}{l}
n \\
2
\end{array}\right)-\overline{\operatorname{stat}}_{X, Y}(\sigma)
$$

It follows that

$$
q^{\left(\begin{array}{c}
n \\
2
\end{array}\right)} P_{n, s, t}^{X, Y}(1 / q)=\bar{P}_{n, s, t}^{X, Y}(q)
$$

It is possible to show that one can prove (1.6) and (1.7) from the recursions (2.4) and (2.7). We shall not give such proofs here, but instead give direct combinatorial proofs of (1.6) and (1.7) which will give us non-recursive descriptions of the statistics $\operatorname{stat}_{X, Y}$ and $\overline{\operatorname{stat}}_{X, Y}$.

\section{Combinatorial Proofs}

In this section, we shall show how to modify the combinatorial proofs of (1.2) and (1.3) found in Hall and Remmel [4] to give combinatorial proofs of (1.6) and (1.7). We start with the proof of (1.6).

Theorem 3.1. Let

$$
P_{n}^{X, Y}(q, x)=\sum_{s \geqslant 0} P_{n, s}^{X, Y}(q) x^{s}:=\sum_{\sigma \in S_{n}} q^{\operatorname{inv}_{X^{c}}(\sigma)+\operatorname{rlmaj}_{X, Y}(\sigma)+y^{c} x \operatorname{coinv}_{X, Y}(\sigma)} x^{\operatorname{des}_{X, Y}(\sigma)},
$$

where

$$
\begin{aligned}
\operatorname{inv}_{X^{c}}(\sigma) & =\sum_{i=1}^{n}\left(\# j \in X^{c} \text { s.t. } j \text { appears to the left of } i \text { and } j>i\right) \\
\operatorname{rlmaj}_{X, Y}(\sigma) & =\sum_{i \in \operatorname{Des}_{X, Y}(\sigma)}(n-i), \text { and } \\
y^{c} x \operatorname{coinv}_{X, Y}(\sigma) & =\sum_{x \in X_{n}}\left(\# z \in Y^{c} \text { s.t. } z \text { appears to the left of } x \text { and } z<x\right) .
\end{aligned}
$$


Then

$$
\begin{aligned}
& P_{n, s}^{X, Y}(q)= \\
& {\left[\left|X_{n}^{c}\right|\right]_{q} ! \sum_{r=0}^{s}(-1)^{s-r} q^{\left(\frac{s-r}{2}\right)}\left[\begin{array}{c}
\left|X_{n}^{c}\right|+r \\
r
\end{array}\right]_{q}\left[\begin{array}{c}
n+1 \\
s-r
\end{array}\right]_{q} \prod_{x \in X_{n}}\left[1+r+\alpha_{X, n, x}+\beta_{Y, n, x}\right]_{q},}
\end{aligned}
$$

where

$$
\begin{aligned}
X_{n} & =X \cap[n], \\
X_{n}^{c} & =\left(X^{c}\right)_{n}=[n]-X,
\end{aligned}
$$

and for any set $A$,

$$
\begin{aligned}
\alpha_{A, n, j} & =\left|A^{c} \cap\{j+1, j+2, \ldots, n\}\right|=|\{z: j<z \leqslant n \& z \notin A\}|, \text { and } \\
\beta_{A, n, j} & =\left|A^{c} \cap\{1,2, \ldots, j-1\}\right|=|\{z: 1 \leqslant z<j \& z \notin A\}| .
\end{aligned}
$$

Proof. The proofs are analogous to those presented in [4], with the addition of $q$-weights on the objects of the sign-reversing involutions.

Let $X, Y, n$, and $s$ be given. For $r$ satisfying $0 \leqslant r \leqslant s$, we define the set of what we call $(n, s, r)^{X, Y}$-configurations. An $(n, s, r)^{X, Y}$-configuration $c$ consists of an array of the numbers $1,2, \ldots, n, r+$ 's, and $(s-r)$-'s, satisfying the following two conditions:

(i) each - is either at the very beginning of the array or immediately follows a number, and

(ii) if $x \in X$ and $y \in Y$ are consecutive numbers in the array, and $x>y$, i.e., if $(x, y)$ forms an $(X, Y)$-descent pair in the underlying permutation, then there must be at least one + between $x$ and $y$.

Note that in an $(n, s, r)^{X, Y}$-configuration, the number of +'s plus the number of -'s equals $s$.

For example, if $X=\{2,3,5,6\}$ and $Y=\{1,3\}$, the following is a $(6,5,3)^{X, Y}$ configuration:

$$
c=5+2-+46+13-.
$$

In this example, the underlying permutation is 524613 .

In general, we will let $c_{1} c_{2} \cdots c_{n}$ denote the underlying permutation of the $(n, s, r)^{X, Y}$ configuration $c$.

Let $C_{n, s, r}^{X, Y}$ be the set of all $(n, s, r)^{X, Y}$-configurations. We claim that

$$
\left|C_{n, s, r}^{X, Y}\right|=\left|X_{n}^{c}\right| !\left(\begin{array}{c}
\left|X_{n}^{c}\right|+r \\
r
\end{array}\right)\left(\begin{array}{c}
n+1 \\
s-r
\end{array}\right) \prod_{x \in X_{n}}\left(1+r+\alpha_{X, n, x}+\beta_{Y, n, x}\right) .
$$

That is, we can construct the $(n, s, r)^{X, Y}$-configurations as follows. First, we pick an order for the elements in $X_{n}^{c}$. This can be done in $\left|X_{n}^{c}\right|$ ! ways. Next, we insert the $r+$ 's. This can be done in $\left(\begin{array}{c}\left|X_{n}^{c}\right|+r \\ r\end{array}\right)$ ways. Next, we insert the elements of $X_{n}=\left\{x_{1}<x_{2}<\cdots<x_{\left|X_{n}\right|}\right\}$ in increasing order. After placing $x_{1}, x_{2}, \ldots, x_{i-1}$, the next element $x_{i}$ can be placed 
- immediately before any of the $\beta_{Y, n, x_{i}}$ elements of $\left\{1,2, \ldots, x_{i-1}\right\}$ that is not in $Y$, or

- immediately before any of the $\alpha_{X, n, x_{i}}$ elements of $\left\{x_{i}+1, x_{i}+2, \ldots, n\right\}$ that is not in $X$, or

- immediately before any of the $r+$ 's, or

- at the very end of the array.

Thus we can place the elements of $X_{n}$ in $\prod_{x \in X_{n}}\left(1+r+\alpha_{X, n, x}+\beta_{Y, n, x}\right)$ ways. Note that although $x_{i}$ might also be in $Y$, and might be placed immediately after some other element of $X_{n}$, condition (ii) is not violated because the elements of $X_{n}$ are placed in increasing order. Finally, since each - must occur either at the very start of the configuration or immediately following a number, we can place the -'s in $\left(\begin{array}{l}n+1 \\ s-r\end{array}\right)$ ways.

Let the $q$-weight $w_{q}(c)$ of an $(n, s, r)^{X, Y}$-configuration $c$ be

$$
(-1)^{s-r} q^{\operatorname{inv}_{X^{c}}(c)+\operatorname{rlmaj}_{X, Y}(c)+y^{c} x \operatorname{coinv}_{X, Y}(c)},
$$

where

$$
\begin{aligned}
\operatorname{inv}_{X^{c}}(c) & =\sum_{i=1}^{n}\left(\# j \in X^{c} \text { s.t. } j \text { appears to the left of } i \text { and } j>i\right), \\
\operatorname{rlmaj}_{X, Y}(c) & =\sum_{i=1}^{n}(\# \text { signs to the left of } i), \text { and } \\
y^{c} x \operatorname{coinv}_{X, Y}(c) & =\sum_{x \in X_{n}}\left(\# z \in Y^{c} \text { s.t. } z \text { appears to the left of } x \text { and } z<x\right)
\end{aligned}
$$

In our example, with $X=\{2,3,5,6\}, Y=\{1,3\}$, and $c$ the $(6,5,3)^{X, Y}$-configuration

$$
5+2-+46+13-,
$$

we have

$$
\begin{aligned}
\operatorname{inv}_{X^{c}}(c) & =0+0+0+0+1+1=2, \\
\operatorname{rlmaj}_{X, Y}(c) & =0+1+3+3+4+4=15, \text { and } \\
y^{c} x \operatorname{coinv}_{X, Y}(c) & =0+0+3+1=4 .
\end{aligned}
$$

The $q$-weight of this configuration is thus $w_{q}(c)=(-1)^{5-3} q^{2+15+4}=q^{21}$.

Next we must show that

$$
\begin{aligned}
& S_{n, s, r}=\sum_{c \in C_{n, S, r}^{X, Y}} w_{q}(c)= \\
& (-1)^{s-r}\left[\left|X_{n}^{c}\right|\right]_{q} !\left[\begin{array}{c}
\left|X_{n}^{c}\right|+r \\
r
\end{array}\right]_{q} q^{\left(\begin{array}{c}
s-r \\
2
\end{array}\right)}\left[\begin{array}{c}
n+1 \\
s-r
\end{array}\right]_{q} \prod_{x \in X_{n}}\left[1+r+\alpha_{X, n, x}+\beta_{Y, n, x}\right]_{q} .
\end{aligned}
$$


We shall use two well-known results to help us prove (3.2). That is, for any sequence $s=s_{1} \cdots s_{n}$ of natural numbers, we let $\operatorname{inv}(s)=\sum_{1 \leqslant i<j \leqslant n} \chi\left(s_{i}>s_{j}\right)$ and $\operatorname{coinv}(\mathrm{s})=$ $\sum_{1 \leqslant i<j \leqslant n} \chi\left(s_{i}<s_{j}\right)$ where for any statement $A, \chi(A)=1$ is $A$ is true and $\chi(A)=0$ if $A$ is false. Then for any $n \geqslant 1$,

$$
\sum_{\sigma \in S_{n}} q^{\operatorname{inv}(\sigma)}=\sum_{\sigma \in S_{n}} q^{\operatorname{coinv}(\sigma)}=[n]_{q} ! .
$$

Similarly we let $\mathcal{R}\left(1^{k} 0^{n-k}\right)$ denote the set of rearrangement of $k 1$ 's and $n-k 0$ 's. Then

$$
\sum_{r \in \mathcal{R}\left(1^{k} 0^{n-k}\right)} q^{\operatorname{inv}(r)}=\left[\begin{array}{l}
n \\
k
\end{array}\right]_{q} .
$$

If we count the inversions caused by the 1 's reading from right to left in any $r \in \mathcal{R}\left(1^{k} 0^{n-k}\right)$, it follows that

$$
\left[\begin{array}{l}
n \\
k
\end{array}\right]_{q}=\sum_{0 \leqslant i_{1} \leqslant \cdots \leqslant i_{k} \leqslant n-k} q^{i_{1}+\cdots+i_{k}},
$$

and if we replace each $i_{s}$ in $(3.5)$ by $j_{s}=i_{s}+s-1$, then it is easy to see that

$$
q^{\left(\begin{array}{c}
k \\
2
\end{array}\right)}\left[\begin{array}{c}
n \\
k
\end{array}\right]_{q}=\sum_{0 \leqslant j_{1}<\cdots<j_{k} \leqslant n-1} q^{j_{1}+\cdots+j_{k}} .
$$

Now consider how we constructed the elements of $C_{n, s, r}^{X, Y}$ above. We first put down a permutation of the elements of $X_{n}^{c}$. Since each inversion among these elements contributes 1 to $\operatorname{inv}_{X^{c}}(c)$, these placements contribute a factor of $\left[\left|X_{n}^{c}\right|\right]_{q} !$ to $S_{n, s, r}$ by (3.3). Next, we insert the $r+$ 's. Since each + contributes 1 for each element of $X_{n}^{c}$ to its right to $\operatorname{rlmaj}_{X, Y}(c)$, the $q$-count over all possible ways of inserting the $r+$ 's into our permutation of $X_{n}^{c}$ is the same as the number of inversions between $r$ 's and $\left|X_{n}^{c}\right| 0$ 's. Thus the insertion of the $r+$ 's contributes a factor of $\left[\begin{array}{c}\left|X_{n}^{c}\right|+r \\ r\end{array}\right]_{q}$ to $S_{n, s, r}$ by (3.4). Next, we insert the elements of $X_{n}=\left\{x_{1}<x_{2}<\cdots<x_{\left|X_{n}\right|}\right\}$ in increasing order. After placing $x_{1}, x_{2}, \ldots, x_{i-1}$, the next element $x_{i}$ can go

- immediately before any of the $\beta_{Y, n, x_{i}}$ elements of $\left\{1,2, \ldots, x_{i-1}\right\}$ that is not in $Y$, or

- immediately before any of the $\alpha_{X, n, x_{i}}$ elements of $\left\{x_{i}+1, x_{i}+2, \ldots, n\right\}$ that is not in $X$, or

- immediately before any of the $r+$ 's, or

- at the very end of the array.

Note that each of the elements counted by $\beta_{Y, n, x_{i}}$ to the left of $x_{i}$ contributes 1 to $y^{c} x \operatorname{coinv}_{X, Y}(c)$, each of the elements counted by $\alpha_{X, n, x_{i}}$ to the left of $x_{i}$ contributes 1 to $\operatorname{inv}_{X^{c}}(c)$, and each of the +'s to the left of $x_{i}$ contributes 1 to $\operatorname{rlmaj}_{X, Y}(c)$. Thus it 
follows that the placement of $x_{i}$ contributes a factor of $1+q+\cdots+q^{r+\alpha_{X, n, x_{i}}+\beta_{Y, n, x_{i}}}=$ $\left[1+r+\alpha_{X, n, x_{i}}+\beta_{Y, n, x_{i}}\right]_{q}$ to $S_{n, s, r}$. Finally we must insert the $(s-r)$-'s. Since each - must occur either at the very start of the configuration or immediately following a number and each - contributes the number of elements of $\{1, \ldots n\}$ that lie to its right to $\operatorname{rlmaj}_{X, Y}(c)$, it follows that the contribution over all such placements to $S_{n, s, r}$ is

$$
\sum_{0 \leqslant j_{1}<\cdots<j_{s-r} \leqslant n} q^{j_{1}+\cdots j_{s-r}}=q^{\left(\frac{s-r}{2}\right)}\left[\begin{array}{c}
n+1 \\
s-r
\end{array}\right]_{q}
$$

by (3.6). Thus we have established that the right-hand side of (3.1) is the sum of the $w_{q}(c)$ over all possible configurations.

We now prove (3.1) by exhibiting a weight-preserving sign-reversing involution $I$ on the set $C_{n, s}^{X, Y}=\bigsqcup_{r=0}^{s} C_{n, s, r}^{X, Y}$, whose fixed points correspond to permutations $\sigma \in S_{n}$ such that $\operatorname{des}_{X, Y}(\sigma)=s$. We say that a sign can be "reversed" if it can be changed from + to - or from - to + without violating conditions (i) and (ii). To apply $I$ to a configuration $c$, we scan from left to right until we find the first sign that can be reversed. We then reverse that sign, and we let $I(c)$ be the resulting configuration. If no signs can be reversed, we set $I(c)=c$.

In our example, with $X=\{2,3,5,6\}, Y=\{1,3\}$, and $c$ the $(6,5,3)^{X, Y}$-configuration

$$
5+2-+46+13-
$$

the first sign we encounter is the + following 5 . This + can be reversed, since 52 is not an $(X, Y)$-descent. Thus $I(c)$ is the configuration shown below:

$$
I(c)=5-2-+46+13-.
$$

It is easy to see that $I(I(c))=c$ in this case, since applying $I$ again we change the following 5 back to a + .

Conditions (i) and (ii) are clearly preserved by the very definition of $I$. It is also clear that $I$ is sign-reversing, since if $I(c) \neq c$, then $I(c)$ either has one more - than $c$, or one fewer - than $c . I$ also preserves the $q$-weight, since $\operatorname{inv}_{X^{c}}(c), \operatorname{rlcomaj}_{X, Y}(c)$, and $y^{c} x \operatorname{coinv}_{X, Y}(c)$ depend only on the underlying permutation and the distribution of signs (without regard to + or - ), neither of which is changed by $I$. To see that $I$ is in fact an involution, we note that the only signs that are not reversible are single + 's occurring in the middle of an $(X, Y)$-descent pair, and +'s that immediately follow another sign. In either case, it is clear that a sign is reversible in a configuration $c$ if and only if the corresponding sign is reversible in $I(c)$. Thus, if a sign is the first reversible sign in $c$, the corresponding sign in $I(c)$ must also be the first reversible sign in $I(c)$. It follows that $I(I(c))=c$ for all $c \in C_{n, s}^{X, Y}$. We therefore have

$$
\sum_{r=0}^{s} \sum_{c \in C_{n, s, r}^{X, Y}} w_{q}(c)=\sum_{r=0}^{s} \sum_{c \in C_{n, s, r}^{X, Y}, I(c)=c} w_{q}(c) .
$$


Now, consider the fixed points of $I$. Suppose that $I(c)=c$. Then $c$ clearly can have no -'s, and thus $r=s$. This implies that the sign associated with the configuration $c$ is positive. It must also be the case that no +'s can be reversed. Thus each of the $s+$ 's must occur singly in the middle of an $(X, Y)$-descent pair. It follows that the underlying permutation has exactly $s(X, Y)$-descents.

Finally, we should observe that if $\sigma=\sigma_{1} \sigma_{2} \cdots \sigma_{n}$ is a permutation with exactly $s$ $(X, Y)$-descents, then we can create a fixed point of $I$ simply by placing a + in the middle of each $(X, Y)$-descent pair.

For example, if $X=\{2,4,6,9\}, Y=\{1,4,7\}, n=9, s=2$, and $\sigma=528941637$, then the corresponding fixed point is

$$
c=5289+4+1637 \text {. }
$$

Note that in such a case, if $\sigma_{i}>\sigma_{i+1}$ is an $X, Y$-descent in $\sigma$, then in the corresponding fixed point $c$ of $I$, we will have a + between $\sigma_{i}$ and $\sigma_{i+1}$. This means that each of $\sigma_{i+1}, \ldots, \sigma_{n}$ will contribute 1 to $\operatorname{rlmaj}_{X, Y}(c)$ for the + between $\sigma_{i}$ and $\sigma_{i+1}$. Hence it follows that

$$
\operatorname{rlmaj}_{X, Y}(c)=\sum_{i \in \operatorname{Des}_{X, Y}(\sigma)}(n-i) .
$$

Thus for any permutation $\sigma \in S_{n}$ with $s X, Y$-descents, the weight $w_{q}(c)$ of the corresponding configuration $c$ which is a fixed point of $I$ is

$$
q^{\operatorname{inv}_{X}(\sigma)+\operatorname{rlmaj}_{X, Y}(\sigma)+y^{c} x \operatorname{coinv}_{X, Y}(\sigma)}
$$

where

$$
\begin{aligned}
\operatorname{inv}_{X^{c}}(\sigma) & =\sum_{i=1}^{n}\left(\# j \in X^{c} \text { s.t. } j \text { appears to the left of } i \text { and } j>i\right) \\
\operatorname{rlmaj}_{X, Y}(\sigma) & =\sum_{i \in \operatorname{Des}_{X, Y}(\sigma)}(n-i), \text { and } \\
y^{c} x \operatorname{coinv}_{X, Y}(\sigma) & =\sum_{x \in X_{n}}\left(\# z \in Y^{c} \text { s.t. } z \text { appears to the left of } x \text { and } z<x\right)
\end{aligned}
$$

as desired.

Our next result shows that the statistics defined in Sections 2 and 3 are in fact the same.

Theorem 3.2. For all $\sigma \in S_{n}$,

$$
\operatorname{stat}_{X, Y}(\sigma)=\operatorname{inv}_{X^{c}}(\sigma)+\operatorname{rlmaj}_{X, Y}(\sigma)+y^{c} x \operatorname{coinv}_{X, Y}(\sigma)
$$

Proof. By definition, it is the case that

$$
\operatorname{stat}_{X, Y}\left(\sigma^{(k)}\right)=\operatorname{stat}_{X, Y}(\sigma)+k .
$$


We will now show that

$$
\begin{aligned}
& \operatorname{inv}_{X^{c}}\left(\sigma^{(k)}\right)+\operatorname{rlmaj}_{X, Y}\left(\sigma^{(k)}\right)+y^{c} x \operatorname{coinv}_{X, Y}\left(\sigma^{(k)}\right) \\
& =\operatorname{inv}_{X^{c}}(\sigma)+\operatorname{rlmaj}_{X, Y}(\sigma)+y^{c} x \operatorname{coinv}_{X, Y}(\sigma)+k,
\end{aligned}
$$

which when combined with the fact that all of the statistics are 0 on $\sigma \in S_{1}$, verifies the theorem. Suppose $\sigma=\sigma_{1} \cdots \sigma_{n} \in S_{n}$ where $\operatorname{des}_{X, Y}(\sigma)=s$ and $\left|Y_{n}^{c}\right|=t$.

Case 1: $n+1 \notin X$ and $\operatorname{des}_{X, Y}\left(\sigma^{(k)}\right)=s-1$

Assume further that $\sigma_{j+1}^{(k)}=n+1$. Inserting $n+1$ at position $j+1$ means that it will contribute $n-j$, the number of elements following $n+1$, to the $\operatorname{inv}_{X^{c}}$ statistic. So we have that

$$
\operatorname{inv}_{X^{c}}\left(\sigma^{(k)}\right)=\operatorname{inv}_{X^{c}}(\sigma)+n-j .
$$

Since we destroyed an $X, Y$-descent at position $j$ we lose a contribution of $n-j$ to the rlmaj $_{X, Y}$ statistic. On the other hand, each of the $k X, Y$-descents preceding $n+1$ will contribute 1 to this statistic. Thus,

$$
\operatorname{rlmaj}_{X, Y}\left(\sigma^{(k)}\right)=\operatorname{rlmaj}_{X, Y}(\sigma)-(n-j)+k .
$$

Since $n+1 \notin X$, we have that

$$
y^{c} x \operatorname{coinv}_{X, Y}\left(\sigma^{(k)}\right)=y^{c} x \operatorname{coinv}_{X, Y}(\sigma) .
$$

Case 2: $n+1 \notin X$ and $\operatorname{des}_{X, Y}\left(\sigma^{(k)}\right)=s$

Assume further that there are $d X, Y$-descents to the left of $n+1$ in $\sigma^{(k)}$. Inserting $n+1$ at a position labeled $k$ means that it will contribute $k-s+s-d=k-d$, the number of elements following $n+1$, to the $\operatorname{inv}_{X^{c}}$ statistic. So we have that

$$
\operatorname{inv}_{X^{c}}\left(\sigma^{(k)}\right)=\operatorname{inv}_{X^{c}}(\sigma)+k-d .
$$

Since we are dealing with a permutation of length $n+1$, each of the $d X, Y$-descents preceding $n+1$ will contribute 1 to the $\operatorname{rlmaj}_{X, Y}$ statistic. Thus,

$$
\operatorname{rlmaj}_{X, Y}\left(\sigma^{(k)}\right)=\operatorname{rlmaj}_{X, Y}(\sigma)+d
$$

Again, since $n+1 \notin X$, we have that

$$
y^{c} x \operatorname{coinv}_{X, Y}\left(\sigma^{(k)}\right)=y^{c} x \operatorname{coinv}_{X, Y}(\sigma) .
$$

Case 3: $n+1 \in X$ and $\operatorname{des}_{X, Y}\left(\sigma^{(k)}\right)=s$

Assume further that there are $d X, Y$-descents to the left of $n+1$ in $\sigma^{(k)}$. Since $n+1 \in X$, we have that

$$
\operatorname{inv}_{X^{c}}\left(\sigma^{(k)}\right)=\operatorname{inv}_{X^{c}}(\sigma) .
$$

Each of the $d X, Y$-descents preceding $n+1$ will contribute 1 to the $\operatorname{rlmaj}_{X, Y}$ statistic. Thus,

$$
\operatorname{rlmaj}_{X, Y}\left(\sigma^{(k)}\right)=\operatorname{rlmaj}_{X, Y}(\sigma)+d
$$


Inserting $n+1$ at a position labeled $k$ means that it will contribute $k-d$, the number of elements preceding $n+1$ that are in $Y_{n}^{c}$, to the statistic $y^{c} x \operatorname{coinv}_{X, Y}$. So we have that

$$
y^{c} x \operatorname{coinv}_{X, Y}\left(\sigma^{(k)}\right)=y^{c} x \operatorname{coinv}_{X, Y}(\sigma)+k-d .
$$

Case 4: $n+1 \in X$ and $\operatorname{des}_{X, Y}\left(\sigma^{(k)}\right)=s+1$

Assume further that there are $d X, Y$-descents to the left of $n+1$ in $\sigma^{(k)}$ and that $\sigma_{j+1}^{(k)}=n+1$. Again, since $n+1 \in X$, we have that

$$
\operatorname{inv}_{X^{c}}\left(\sigma^{(k)}\right)=\operatorname{inv}_{X^{c}}(\sigma) .
$$

However, since we have created an $X, Y$-descent at position $j+1$ we gain a contribution of $n+1-(j+1)=n-j$ to the $\operatorname{rlmaj}_{X, Y}$ statistic. On the other hand, each of the $d$ $X, Y$-descents preceding $n+1$ will also contribute 1 to this statistic. Thus,

$$
\operatorname{rlmaj}_{X, Y}\left(\sigma^{(k)}\right)=\operatorname{rlmaj}_{X, Y}(\sigma)+d+(n-j) .
$$

Inserting $n+1$ at a position labeled $k$ means that it will contribute $j-(n-k)-d$, the number of elements preceding $n+1$ that are in $Y_{n}^{c}$, to the statistic $y^{c} x \operatorname{coinv}_{X, Y}$. So we have that

$$
y^{c} x \operatorname{coinv}_{X, Y}\left(\sigma^{(k)}\right)=y^{c} x \operatorname{coinv}_{X, Y}(\sigma)+j-(n-k)-d .
$$

In each case we see that inserting $n+1$ into the position labeled with a $k$ contributes $k$ to each statistic and thus they are equivalent.

Next we consider the proof of (1.7).

Theorem 3.3. Let

$$
\bar{P}_{n}^{X, Y}(q, x)=\sum_{s \geqslant 0} \bar{P}_{n, s}^{X, Y}(q) x^{s}:=\sum_{\sigma \in S_{n}} q^{\operatorname{coinv}_{X^{c}}(\sigma)+\operatorname{rlcomaj}_{X, Y}(\sigma)-y^{c} x \operatorname{coinv}(\sigma)} x^{\operatorname{des}_{X, Y}(\sigma)}
$$

where

$$
\begin{aligned}
\operatorname{coinv}_{X^{c}}(\sigma) & =\sum_{i=1}^{n}\left(\# j \in X^{c} \text { s.t. } j \text { appears to the left of } i \text { and } j<i\right) \\
\operatorname{rlcomaj}_{X, Y}(\sigma) & =\sum_{i \notin D e s_{X, Y}(\sigma), \sigma_{i} \in X_{n}}(n-i), \text { and } \\
y^{c} x \operatorname{coinv}_{X, Y}(\sigma) & =\sum_{x \in X_{n}}\left(\# z \in Y^{c} \text { s.t. } z \text { appears to the left of } x \text { and } z<x\right) .
\end{aligned}
$$

Then

$$
\begin{aligned}
\bar{P}_{n, s}^{X, Y}(q)= & {\left[\left|X_{n}^{c}\right|\right]_{q} ! \sum_{r=0}^{\left|X_{n}\right|-s}\left((-1)^{\left|X_{n}\right|-s-r} q^{\left(\left|X_{n}\right|-s-r\right.}\right)\left[\begin{array}{c}
\left|X_{n}^{c}\right|+r \\
r
\end{array}\right]_{q}\left[\begin{array}{c}
n+1 \\
\left|X_{n}\right|-s-r
\end{array}\right]_{q} } \\
& \left.\prod_{x \in X_{n}}\left[r+\beta_{X, n, x}-\beta_{Y, n, x}\right]_{q}\right)
\end{aligned}
$$


where

$$
\begin{aligned}
& X_{n}=X \cap[n], \\
& X_{n}^{c}=\left(X^{c}\right)_{n}=[n]-X,
\end{aligned}
$$

and

$$
\begin{aligned}
& \beta_{X, n, j}=\left|X^{c} \cap\{1,2, \ldots, j-1\}\right|=|\{z: 1 \leqslant z<j \& z \notin X\}| \text { and } \\
& \beta_{Y, n, j}=\left|Y^{c} \cap\{1,2, \ldots, j-1\}\right|=|\{z: 1 \leqslant z<j \& z \notin Y\}| .
\end{aligned}
$$

Proof. Let $X, Y, n$, and $s$ be given. For $r$ satisfying $0 \leqslant r \leqslant\left|X_{n}\right|-s$, an $\overline{(n, s, r)}^{X, Y}$ configuration consists of an array of the numbers $1,2, \ldots, n, r+$ 's, and $\left(\left|X_{n}\right|-s-r\right)$-'s, satisfying the following three conditions:

(i) each - is either at the very beginning of the array or immediately follows a number,

(ii) if $c_{i} \in X, 1 \leqslant i<n$, and $\left(c_{i}, c_{i+1}\right)$ is not an $(X, Y)$-descent pair of the underlying permutation, then there must be at least one + between $c_{i}$ and $c_{i+1}$, and

(iii) if $c_{n} \in X$, then at least one + must occur to the right of $c_{n}$.

Note that in an $\overline{(n, s, r)}^{X, Y}$-configuration, the number of +'s plus the number of -'s equals $\left|X_{n}\right|-s$.

For example, if $X=\{2,3,6\}$ and $Y=\{1,2,5\}$, then the following is a $\overline{(6,1,1)}^{X, Y}$ configuration:

$$
c=213+6-54 \text {. }
$$

Let $\bar{C}_{n, s, r}^{X, Y}$ be the set of all $\overline{(n, s, r)}^{X, Y}$-configurations. Then we claim that

$$
\left|\bar{C}_{n, s, r}^{X, Y}\right|=\left|X_{n}^{c}\right| !\left(\begin{array}{c}
\left|X_{n}^{c}\right|+r \\
r
\end{array}\right)\left(\begin{array}{c}
n+1 \\
\left|X_{n}\right|-s-r
\end{array}\right) \prod_{x \in X_{n}}\left(r+\beta_{X, n, x}-\beta_{Y, n, x}\right) .
$$

That is, we can construct the $\overline{(n, s, r)}^{X, Y}$-configurations as follows. First, we pick an order for the elements in $X_{n}^{c}$. This can be done in $\left|X_{n}^{c}\right|$ ! ways. Next, we insert the $r$ +'s. This can be done in $\left(\begin{array}{c}\left|X_{n}^{c}\right|+r \\ r\end{array}\right)$ ways. Next, we insert the elements of $X_{n}=\left\{x_{1}<\right.$ $\left.x_{2}<\cdots<x_{\left|X_{n}\right|}\right\}$ in increasing order. We can place $x_{1}$ in $r+\beta_{X, n, x_{1}}-\beta_{Y, n, x_{1}}$ ways, since $x_{1}$ can either go immediately before any of the $r+$ 's or immediately before any of the $x_{1}-1-\beta_{Y, n, x_{1}}=\beta_{X, n, x_{1}}-\beta_{Y, n, x_{1}}$ elements of $Y$ which are less than $x_{1}$. We note here that $\beta_{X, n, x_{i}}=x_{i}-i$ for all $i, 1 \leqslant i \leqslant\left|X_{n}\right|$. There are now two cases to consider for the placement of $x_{2}$.

Case 1. $x_{1}$ was placed immediately in front of some element of $y \in Y$. In this case, $x_{2}$ cannot be placed immediately in front of $y$, since this would violate condition (ii). 
$x_{2}$ can be placed before any + or immediately in front of any element of $Y$ which is less than $x_{2}$, except $y$. Hence, $x_{2}$ can be placed in

$$
\begin{aligned}
r+x_{2}-1-\beta_{Y, n, x_{2}}-1 & =r+x_{2}-2-\beta_{Y, n, x_{2}} \\
& =r+\beta_{X, n, x_{2}}-\beta_{Y, n, x_{2}}
\end{aligned}
$$

ways.

Case 2. $x_{1}$ was placed immediately before a + . In this case, $x_{2}$ cannot be placed immediately before the same + , since again we would violate condition (ii). $x_{2}$ can be placed immediately before any of the other +'s or immediately before any element of $Y$ which is less than $x_{2}$. Hence $x_{2}$ can be placed in

$$
\begin{aligned}
r-1+x_{2}-1-\beta_{Y, n, x_{2}} & =r+x_{2}-2-\beta_{Y, n, x_{2}} \\
& =r+\beta_{X, n, x_{2}}-\beta_{Y, n, x_{2}}
\end{aligned}
$$

ways.

In general, having placed $x_{1}, x_{2}, \ldots, x_{i-1}$, we cannot place $x_{i}$ immediately before some $y \in Y, y<x_{i}$, which earlier had an element of $\left\{x_{1}, x_{2}, \ldots, x_{i-1}\right\}$ placed before it. Similarly, we cannot place $x_{i}$ immediately before any + which earlier had an element of $\left\{x_{1}, x_{2}, \ldots, x_{i-1}\right\}$ placed before it. It then follows that there are

$$
\begin{aligned}
r+x_{i}-1-\beta_{Y, n, x_{i}}-(i-1) & =r+x_{i}-i-\beta_{Y, n, x_{i}} \\
& =r+\beta_{X, n, x_{i}}-\beta_{Y, n, x_{i}}
\end{aligned}
$$

ways to place $x_{i}$. Thus, there are total of $\prod_{x \in X_{n}}\left(r+\beta_{X, n, x_{i}}-\beta_{Y, n, x_{i}}\right)$ ways to place $x_{1}, x_{2}, \ldots, x_{\left|X_{n}\right|}$, given our placement of the elements of $X_{n}^{c}$. Finally, we can place the -'s in $\left(\begin{array}{c}n+1 \\ \left|X_{n}\right|-s-r\end{array}\right)$ ways.

Now suppose that $c=c_{1} \cdots c_{n}$ is a configuration in $\bar{C}_{n, s, r}^{X, Y}$. We define the $q$-weight $\bar{w}_{q}(c)$ of an $\overline{(n, s, r)}^{X, Y}$-configuration $c$ to be

$$
(-1)^{\left|X_{n}\right|-s-r} q^{\operatorname{coinv}_{X^{c}}(c)+\operatorname{rlcomaj}_{X, Y}(c)-y^{c} x \operatorname{coinv}_{X, Y}(c)},
$$

where

$$
\begin{aligned}
\operatorname{coinv}_{X^{c}}(c) & =\sum_{i=1}^{n}\left(\# j \in X^{c} \text { s.t. } j \text { appears to the left of } i \text { and } j<i\right) \\
\operatorname{rlcomaj}_{X, Y}(c) & =\sum_{i=1}^{n}(\# \text { signs to the left of } i), \text { and } \\
y^{c} x \operatorname{coinv}_{X, Y}(c) & =\sum_{x \in X_{n}}\left(\# z \in Y^{c} \text { s.t. } z \text { appears to the left of } x \text { and } z<x\right) .
\end{aligned}
$$


In our example, with $X=\{2,3,4\}, Y=\{1,3,5\}$, and $c$ the $\overline{(6,0,3)}^{X, Y}$-configuration

$$
2+5413++6
$$

we have,

$$
\begin{aligned}
\operatorname{coinv}_{X^{c}}(c) & =3, \\
\operatorname{rlcomaj}_{X, Y}(c) & =7, \text { and } \\
y^{c} x \operatorname{coinv}_{X, Y}(c) & =2 .
\end{aligned}
$$

The $q$-weight of this configuration is thus $\bar{w}_{q}(c)=(-1)^{3-0-3} q^{3+7-2}=q^{8}$.

Note that the definition of $\operatorname{rlcomaj}_{X, Y}$ is identical to that of $\operatorname{rlmaj}_{X, Y}$ of Theorem 3.1. However, because the signs play a different role here, it will not be the case that rlmaj ${ }_{X, Y}$ and $\operatorname{rlcomaj}_{X, Y}$ reduce to the same statistics on $S_{n}$.

Let

$$
\bar{S}_{n, s, r}=\sum_{c \in \bar{C}_{n, s, r}^{X, Y}} \bar{w}_{q}(c) .
$$

We must show that

$$
\begin{aligned}
\bar{S}_{n, s, r}= & (-1)^{\left|X_{n}\right|-s-r}\left[\left|X_{n}^{c}\right|\right]_{q} !\left[\begin{array}{c}
\left|X_{n}^{c}\right|+r \\
r
\end{array}\right]_{q} q^{\left(\begin{array}{c}
\left|X_{n}\right|-s-r \\
2
\end{array}\right)}\left[\begin{array}{c}
n+1 \\
\left|X_{n}\right|-s-r
\end{array}\right]_{q} . \\
& \prod_{x \in X_{n}}\left[r+\beta_{X, n, x_{i}}-\beta_{Y, n, x}\right]_{q} .
\end{aligned}
$$

Now consider how we constructed the elements of $\bar{C}_{n, s, r}^{X, Y}$ above. We first put down a permutation of the elements of $X_{n}^{c}$. Since each coinversion among these elements contributes 1 to $\operatorname{coinv}_{X^{c}}(c)$, these elements contribute a factor of $\left[\left|X_{n}^{c}\right|\right]_{q}$ ! to $\bar{S}_{n, s, r}$. Next, we put down the $r+$ 's. Since each + contributes 1 for each element of $X_{n}^{c}$ to its right to $\operatorname{rlcomaj}_{X, Y}(c)$, the $q$-count over all possible ways of inserting the $r+$ 's into our permutation of $X_{n}^{c}$ is the same as the number of inversions between $r$ 1's and $\left|X_{n}^{c}\right| 0$ 's. Thus the insertion of the $r+$ 's contributes a factor of $\left[\begin{array}{c}\left|X_{n}^{c}\right|+r \\ r\end{array}\right]_{q}$ to $\bar{S}_{n, s, r}$. Next, we insert the elements of $X_{n}=\left\{x_{1}<x_{2}<\cdots<x_{\left|X_{n}\right|}\right\}$ in increasing order. Each $x_{i}$ must go either before a + or before an element $y \in Y$ such that $y<x_{i}$. However, having placed $x_{1}, x_{2}, \ldots, x_{i-1}$, we cannot place $x_{i}$ immediately before some $y \in Y, y<x_{i}$, which earlier had an element of $\left\{x_{1}, x_{2}, \ldots, x_{i-1}\right\}$ placed before it. Similarly, we cannot place $x_{i}$ immediately before any + which earlier had an element of $\left\{x_{1}, x_{2}, \ldots, x_{i-1}\right\}$ placed before it. So the number of ways to place $x_{i}$ is the difference between the sum of the number of +'s and the number of elements smaller than $x_{i}$ which are in $Y$, and the number of $x \in X$ already placed, which is $i-1$. This difference is $r+x_{i}-1-\beta_{Y, n, x_{i}}-(i-1)=r+\beta_{X, n, x_{i}}-\beta_{Y, n, x_{i}}$. Now when we place $x_{i}$, each + to the left of $x_{i}$ contributes 1 to $\operatorname{rlcomaj}_{X, Y}(c)$, each $z \in X^{c}, z<x_{i}$, to the left of $x_{i}$ contributes 1 to $\operatorname{coinv}_{X^{c}}(c)$, and each $z \in Y^{c}, z<x_{i}$, contributes 1 to $y^{c} x \operatorname{coinv}_{X, Y}(\mathrm{c})$. The key here is to realize that the difference between the number of 
$y \in Y, y<x_{i}$ to the left of $x_{i}$ and the number of $x \in X, x<x_{i}$ to the left of $x_{i}$ is equal to the difference between the number of $z \in X_{n}^{c}, z<x_{i}$ to the left of $x_{i}$ and the number of $z \in Y_{n}^{c}, y<x_{i}$ to the left of $x_{i}$. In any rate, when we place $x_{i}$, we effectively get a factor of $q$ for each + and each element $y \in Y$ satisfying $y<x_{i}$ which lies to the left of $x_{i}$, and a factor of $q^{-1}$ for each element $x \in X$ satisfying $x<x_{i}$ which lies to the left of $x_{i}$. Thus the net effect is that we have a factor of $q$ for each place before the position of $x_{i}$ which was a possible position where we could have placed $x_{i}$. By our argument above, there are exactly $r+\beta_{X, n, x_{i}}-\beta_{Y, n, x_{i}}$ positions so that the contribution over all possible placements of $x_{i}$ at this stage is $1+q+\cdots q^{r+\beta_{X, n, x_{i}}-\beta_{Y, n, x_{i}}-1}=\left[r+\beta_{X, n, x_{i}}-\beta_{Y, n, x_{i}}\right]_{q}$. Thus the total contribution from the placements of elements in $X_{n}$ is $\prod_{x \in X_{n}}\left[r+\beta_{X, n, x}-\beta_{Y, n, x}\right]_{q}$. Finally we must insert the $\left(\left|X_{n}\right|-s-r\right)$-'s. In this case, we can argue exactly in the proof of Theorem 3.1, that all possible insertions contribute a factor of

$$
\left.\sum_{0 \leqslant j_{1}<\cdots<j_{\left|X_{n}\right|-s-r} \leqslant n} q^{j_{1}+\cdots j_{\left|X_{n}\right|-s-r}}=q^{\left(\left|X_{n}\right|-s-r\right.}\right)\left[\begin{array}{c}
n+1 \\
\left|X_{n}\right|-s-r
\end{array}\right]_{q}
$$

to $\bar{S}_{n, s, r}$. Thus we have established that the right-hand side of (3.9) is the sum of the $\bar{w}_{q}(c)$ over all possible configurations.

We now prove the theorem by exhibiting a sign-reversing involution $I$ on the set $\bar{C}_{n, s}^{X, Y}=\bigsqcup_{r=0}^{\left|X_{n}\right|-s} \bar{C}_{n, s, r}^{X, Y}$ whose fixed points correspond to permutations $\sigma \in S_{n}$ such that $\operatorname{des}_{X, Y}(\sigma)=s$. We define $I$ exactly as in the proof of Theorem 3.1. That is, we scan from left to right and reverse the first sign that we can reverse without violating conditions (i)-(iii).

For example, suppose $X=\{2,3,4\}, Y=\{1,3,5\}$, and we have the $\overline{(6,0,2)}^{X, Y}$. configuration

$$
c=213+6-54+.
$$

We cannot reverse the + following 3 without violating condition (ii), since $3 \in X$ and 36 is not an $(X, Y)$-descent. Thus, we reverse the - following 6 to get

$$
I(c)=213+6+54+.
$$

It is clear that $\bar{w}_{q}(c)= \pm \bar{w}_{q}(I(c))$, since $\operatorname{coinv}_{X^{c}}(c)=\operatorname{coinv}_{X^{c}}(I(c)), \operatorname{rlcomaj}_{X, Y}(c)=$ $\operatorname{rlcomaj}_{X, Y}(I(c))$, and $y^{c} x \operatorname{coinv}_{X, Y}(c)=y^{c} x \operatorname{coinv}_{X, Y}(I(c))$. If $I(c) \neq c$, then $I(c)$ either has one more - than $c$, or one fewer - than $c$, and so $I$ is sign-reversing weight preserving involution.

It follows that

$$
\sum_{r=0}^{\left|X_{n}\right|-s} \sum_{c \in \bar{C}_{n, s, r}^{X, Y}} \bar{w}_{q}(c)=\sum_{r=0}^{\left|X_{n}\right|-s} \sum_{c \in \bar{C}_{n, s, r}^{X, Y}, I(c)=c} \bar{w}_{q}(c) .
$$

Now, consider a fixed point $c$ of $I$. As in the proof of Theorem 3.1, $c$ can have no -'s, and thus $r=\left|X_{n}\right|-s$ and thus $w(c)$ is positive. No string of multiple +'s can occur, since the first + in such a string could be reversed. Thus, each of the $\left(\left|X_{n}\right|-s\right)+$ 's appears singly, and must either 
- immediately follow some $c_{i} \in X, 1 \leqslant i<n$, such that $\left(c_{i}, c_{i+1}\right)$ is not an $(X, Y)$ descent pair of the underlying permutation, or

- immediately follow $c_{n} \in X$.

Thus $\left|X_{n}\right|-s$ elements of $X_{n}$ immediately precede a + that cannot be reversed, and are thus not the tops of $(X, Y)$-descent pairs. It follows that each of the remaining $s$ elements of $X_{n}$ do not immediately precede a + , and as such each must be the top of an $(X, Y)$ descent pair. Thus the underlying permutation $c_{1} c_{2} \cdots c_{n}$ has exactly $s(X, Y)$-descents.

Again, we observe that if $\sigma_{1} \sigma_{2} \cdots \sigma_{n}$ is a permutation with exactly $s(X, Y)$-descents, then we can create a fixed point of $I$ by inserting a + after every element of $X_{n}$ that is not the top of an $(X, Y)$-descent pair. For example, if $X=\{2,3,4,6,8,9\}, Y=\{1,2,3,5\}$, $n=9, s=4$, and $\sigma=958621437$, then the corresponding configuration would be

$$
958+62143+7
$$

Finally, observe that if such a $\sigma$ corresponds to a fixed point of $I$, then for every pair $\left(\sigma_{i}, \sigma_{i+1}\right)$ such that $\sigma_{i} \in X_{n}$ and $\left(\sigma_{i}, \sigma_{i+1}\right)$ is not a $(X, Y)$-descent pair, the + between $\sigma_{i}$ and $\sigma_{i+1}$ in the corresponding configuration contributes 1 for each of $\sigma_{i+1}, \ldots, \sigma_{n}$. It then follows that

$$
\operatorname{rlcomaj}_{X, Y}(c)=\sum_{\sigma_{i} \in X_{n}, i \notin D e s X, Y(\sigma)}(n-i)=\operatorname{rlcomaj}_{X, Y}(\sigma) \text {. }
$$

For any permutation $\sigma$, we now have

$$
\begin{aligned}
& \operatorname{stat}(\sigma)+\operatorname{coinv}_{X^{c}}(\sigma)+\operatorname{rlcomaj}_{X, Y}(\sigma)-y^{c} x \operatorname{coinv}(\sigma)= \\
& \operatorname{inv}_{X^{c}}(\sigma)+\operatorname{rlmaj}_{X, Y}(\sigma)+y^{c} x \operatorname{coinv}(\sigma)+ \\
& \operatorname{coinv}_{X^{c}}(\sigma)+\operatorname{rlcomaj}_{X, Y}(\sigma)-y^{c} x \operatorname{coinv}(\sigma)= \\
& \operatorname{inv}_{X^{c}}(\sigma)+\operatorname{coinv}_{X^{c}}(\sigma)+\operatorname{rlmaj}_{X, Y}(\sigma)+\operatorname{rlcomaj}_{X, Y}(\sigma) .
\end{aligned}
$$

But for any $\sigma=\sigma_{1} \cdots \sigma_{n}, i \in\{1, \ldots, n\}$, and $X, Y \subseteq \mathbb{N}$, it will be that case that if $\sigma_{i} \in X_{n}^{c}$, then we get a contribution of 1 to $\operatorname{inv}_{X^{c}}(\sigma)+\operatorname{coinv}_{X^{c}}(\sigma)$ for each $\sigma_{j}$ with $j>i$ so that

$$
\operatorname{inv}_{X^{c}}(\sigma)+\operatorname{coinv}_{X^{c}}(\sigma)=\sum_{\sigma_{i} \in X_{n}^{c}}(n-i) .
$$

On the other hand, it easy to see from our definition that

$$
\operatorname{rlmaj}_{X, Y}(\sigma)+\operatorname{rlcomaj}_{X, Y}(\sigma)=\sum_{\sigma_{i} \in X_{n}}(n-i) .
$$

Thus

$$
\operatorname{inv}_{X^{c}}(\sigma)+\operatorname{coinv}_{X^{c}}(\sigma)+\operatorname{rlmaj}_{X, Y}(\sigma)+\operatorname{rlcomaj}_{X, Y}(\sigma)=\sum_{i=1}^{n}(n-i)=\left(\begin{array}{l}
n \\
2
\end{array}\right) .
$$


It thus follows that

$$
\operatorname{coinv}_{X^{c}}(\sigma)+\operatorname{rlcomaj}_{X, Y}(\sigma)-y^{c} x \operatorname{coinv}(\sigma)=\left(\begin{array}{l}
n \\
2
\end{array}\right)-\operatorname{stat}(\sigma)=\overline{\operatorname{stat}}(\sigma) .
$$

Thus we have proved the following theorem.

Theorem 3.4. For all permutations $\sigma$,

$$
\overline{\operatorname{stat}}(\sigma)=\operatorname{coinv}_{X^{c}}(\sigma)+\operatorname{rlcomaj}_{X, Y}(\sigma)-y^{c} x \operatorname{coinv}(\sigma) .
$$

Remark 3.5. Note that we have shown that $\operatorname{stat}_{X, Y}=\operatorname{inv}_{X^{c}}+\operatorname{rlmaj}_{X, Y}+y^{c} x \operatorname{coinv}_{X, Y}$ is a Mahonian statistic for all $X$ and $Y$, and interpolates between inv, rlmaj, and coinv, in the sense that $\operatorname{stat}_{\emptyset, \emptyset}(\sigma)=\operatorname{inv}(\sigma), \operatorname{stat}_{\mathbb{N}, \mathbb{N}}(\sigma)=\operatorname{rlmaj}(\sigma)$, and $\operatorname{stat}_{\mathbb{N}, \emptyset}(\sigma)=\operatorname{coinv}(\sigma)$ Similarly, $\overline{\operatorname{stat}}_{X, Y}=\operatorname{coinv}_{X^{c}}+\operatorname{rlcomaj}_{X, Y}-y^{c} x \operatorname{coinv}_{X, Y}$ is a Mahonian statistic for all $X$ and $Y$, and interpolates between coinv and rlcomaj, in the sense that $\overline{\operatorname{stat}}_{\emptyset, \emptyset}(\sigma)=\operatorname{coinv}(\sigma)$ and $\overline{\operatorname{stat}}_{\mathbb{N}, \mathbb{N}}(\sigma)=\operatorname{rlcomaj}(\sigma)$.

\section{Applications}

Note that the results of Sections 2 and 3 show that for all $X, Y \subseteq \mathbb{N}$ and $n \geqslant 1$,

$$
\bar{P}_{n, s}^{X, Y}(q)=q^{\left(\begin{array}{c}
n \\
2
\end{array}\right)} P_{n, s}^{X, Y}(1 / q) .
$$

But then by Theorem 3.1, we have that

$$
\begin{aligned}
\bar{P}_{n, s}^{X, Y}(q)= & q^{\left(\begin{array}{c}
n \\
2
\end{array}\right)}\left[\left|X_{n}^{c}\right|\right]_{1 / q} ! \sum_{r=0}^{s}\left((-1)^{s-r} q^{-\left(\begin{array}{c}
s-r \\
2
\end{array}\right)}\left[\begin{array}{c}
\left|X_{n}^{c}\right|+r \\
r
\end{array}\right]_{1 / q}\left[\begin{array}{c}
n+1 \\
s-r
\end{array}\right]_{1 / q}\right. \\
& \left.\prod_{x \in X_{n}}\left[1+r+\alpha_{X, n, x}+\beta_{Y, n, x}\right]_{1 / q}\right)
\end{aligned}
$$

But then we can use the identities

$$
\begin{aligned}
{[n]_{1 / q} } & =q^{-(n-1)}[n]_{q}, \\
{[n]_{1 / q} ! } & =q^{-\left(\begin{array}{c}
n \\
2
\end{array}\right)}\left([n]_{q} !\right), \text { and } \\
{\left[\begin{array}{c}
n \\
k
\end{array}\right]_{1 / q} } & =q^{\left(\begin{array}{c}
k \\
2
\end{array}\right)+\left(\begin{array}{c}
n-k \\
2
\end{array}\right)-\left(\begin{array}{c}
n \\
2
\end{array}\right)}\left[\begin{array}{l}
n \\
k
\end{array}\right]_{q}
\end{aligned}
$$

to rewrite $(4.1)$ as

$$
\begin{aligned}
\bar{P}_{n, s}^{X, Y}(q)= & {\left[\left|X_{n}^{c}\right|\right]_{q} ! \sum_{r=0}^{s}\left((-1)^{s-r} q^{A}\left[\begin{array}{c}
\left|X_{n}^{c}\right|+r \\
r
\end{array}\right]_{q}\left[\begin{array}{c}
n+1 \\
s-r
\end{array}\right]_{q} .\right.} \\
& \left.\prod_{x \in X_{n}} q^{-\left(r+\alpha_{X, n, x}+\beta_{Y, n, x}\right)}\left[1+r+\alpha_{X, n, x}+\beta_{Y, n, x}\right]_{q}\right)
\end{aligned}
$$


where

$$
\begin{aligned}
A= & \left(\begin{array}{c}
n \\
2
\end{array}\right)-\left(\begin{array}{c}
\left|X_{n}^{c}\right| \\
2
\end{array}\right)-\left(\begin{array}{c}
s-r \\
2
\end{array}\right)+\left(\begin{array}{c}
r \\
2
\end{array}\right)+\left(\begin{array}{c}
\left|X_{n}^{c}\right| \\
2
\end{array}\right)-\left(\begin{array}{c}
\left|X_{n}^{c}\right|+r \\
2
\end{array}\right)+ \\
& \left(\begin{array}{c}
s-r \\
2
\end{array}\right)+\left(\begin{array}{c}
n+1-(s-r) \\
2
\end{array}\right)-\left(\begin{array}{c}
n+1 \\
2
\end{array}\right) \\
= & \left(\begin{array}{c}
r \\
2
\end{array}\right)-\left(\begin{array}{c}
\left|X_{n}^{c}\right|+r \\
2
\end{array}\right)+\left(\begin{array}{c}
n+1-(s-r) \\
2
\end{array}\right)-n \\
= & \left(\begin{array}{c}
r \\
2
\end{array}\right)-\left(\left(\begin{array}{c}
\left|X_{n}^{c}\right| \\
2
\end{array}\right)+\left(\begin{array}{c}
r \\
2
\end{array}\right)+r\left|X_{n}^{c}\right|\right)+\left(\begin{array}{c}
n+1-(s-r) \\
2
\end{array}\right)-n \\
= & \left.\left(\begin{array}{c}
n+1-(s-r) \\
2
\end{array}\right)-\left(\begin{array}{c}
\left|X_{n}^{c}\right| \\
2
\end{array}\right)-n-r \mid X_{n}^{c}\right] .
\end{aligned}
$$

We can then factor out a $q^{r}$ for each term in the product to get a factor of $q^{-r\left|X_{n}\right|}$ which can be combined with the factor $q^{-r\left|X_{n}^{c}\right|}$ to obtain a factor $q^{-r\left(\left|X_{n}\right|+\left|X_{n}^{c}\right|\right)}=q^{-r n}$. It then follows that

$$
\begin{aligned}
& \bar{P}_{n, s}^{X, Y}(q)= \\
& {\left[\left|X_{n}^{c}\right|\right]_{q} ! \sum_{r=0}^{s}(-1)^{s-r} q^{\left(\begin{array}{c}
n+1-(s-r) \\
2
\end{array}\right)-\left(\begin{array}{c}
\left|X_{n}^{c}\right| \\
2
\end{array}\right)-(r+1) n}\left[\begin{array}{c}
\left|X_{n}^{c}\right|+r \\
r
\end{array}\right]_{q}\left[\begin{array}{c}
n+1 \\
s-r
\end{array}\right]_{q} \times} \\
& \quad \prod_{x \in X_{n}} q^{-\left(\alpha_{X, n, x}+\beta_{Y, n, x}\right)}\left[1+r+\alpha_{X, n, x}+\beta_{Y, n, x}\right]_{q}
\end{aligned}
$$

Comparing (4.3) and (3.9), we have proved the following identity.

Theorem 4.1. For all $X, Y \subseteq \mathbb{N}$ and $1 \leqslant s \leqslant n$,

$$
\begin{aligned}
& {\left[\left|X_{n}^{c}\right|\right]_{q} ! \sum_{r=0}^{s}\left((-1)^{s-r} q^{\left(\begin{array}{c}
n+1-(s-r) \\
2
\end{array}\right)-\left(\begin{array}{c}
\left|X_{n}^{c}\right| \\
2
\end{array}\right)-(r+1) n}\left[\begin{array}{c}
\left|X_{n}^{c}\right|+r \\
r
\end{array}\right]_{q}\left[\begin{array}{c}
n+1 \\
s-r
\end{array}\right]_{q} .\right.} \\
& \left.\prod_{x \in X_{n}} q^{-\left(\alpha_{X, n, x}+\beta_{Y, n, x}\right)}\left[1+r+\alpha_{X, n, x}+\beta_{Y, n, x}\right]_{q}\right) \\
& =\left[\left|X_{n}^{c}\right|\right]_{q} ! \sum_{r=0}^{\left|X_{n}\right|-s}\left((-1)^{\left|X_{n}\right|-s-r} q^{\left(\left|X_{n}\right|-s-r\right.}\right)\left[\begin{array}{c}
\left|X_{n}^{c}\right|+r \\
r
\end{array}\right]_{q}\left[\begin{array}{c}
n+1 \\
\left|X_{n}\right|-s-r
\end{array}\right]_{q} . \\
& \left.\prod_{x \in X_{n}}\left[r+\beta_{X, n, x}-\beta_{Y, n, x}\right]_{q}\right)
\end{aligned}
$$

For some sets $X$ and $Y$ this identity can be rewritten in terms of hypergeometric series; hence we obtain combinatorial proofs of identities such as the following, which is a special case of an integral form of a transformation of Karlsson-Minton type basic hypergeometric 
series due to Gasper [2]. Here we employ the notation of basic hypergeometric series, in particular

$$
{ }_{m+1} \phi_{m}\left[\begin{array}{ccccc}
a_{0}, & a_{1}, & a_{2}, & \ldots, & a_{m} \\
& b_{1}, & b_{2}, & \ldots, & b_{m}
\end{array} ; q, x\right]:=\sum_{r=0}^{\infty} \frac{\left(a_{0} ; q\right)_{r}\left(a_{1} ; q\right)_{r}\left(a_{2} ; q\right)_{r} \cdots\left(a_{m} ; q\right)_{r}}{(q ; q)_{r}\left(b_{1} ; q\right)_{r}\left(b_{2} ; q\right)_{r} \cdots\left(b_{m} ; q\right)_{r}} x^{r}
$$

Corollary 4.2. Let $u=\left(u_{1}, \ldots, u_{k}\right)$ be a weakly increasing array of non-negative integers, and $v=\left(v_{1}, \ldots, v_{k}\right)$ an array of positive integers. Then for $n \geqslant \sum_{i=1}^{k} v_{i}+\max \left\{u_{i}+v_{i}: 1 \leqslant\right.$ $i \leqslant k\}-u_{1}$, we have

$$
\begin{aligned}
& q^{\left(\begin{array}{c}
n+1 \\
2
\end{array}\right)}\left(q^{-(a+s)} ; q\right)_{a}\left(q^{-\left(u_{1}+v_{1}+s\right)} ; q\right)_{v_{1}} \cdots\left(q^{-\left(u_{k}+v_{k}+s\right)} ; q\right)_{v_{k}} \\
& \times{ }_{k+2} \phi_{k+1}\left[\begin{array}{ccccc}
q^{-(n+1)}, & q^{-s}, & q^{-\left(s+u_{1}\right)}, & \ldots, & q^{-\left(s+u_{k}\right)} \\
& q^{-(s+a)}, & q^{-\left(s+u_{1}+v_{1}\right)}, & \ldots, & \left.q^{-\left(s+u_{k}+v_{k}\right)}, ; q, q\right]=
\end{array}\right]= \\
& (-1)^{n}\left(q^{n+1-a-s} ; q\right)_{a}\left(q^{n+1-u_{1}-v_{1}-s} ; q\right)_{v_{1}} \cdots\left(q^{n+1-u_{k}-v_{k}-s} ; q\right)_{v_{k}} \\
& \times{ }_{k+2} \phi_{k+1}\left[\begin{array}{ccccc}
q^{-(n+1)}, & q^{-(n-a-s)}, & q^{-\left(n-u_{1}-v_{1}-s\right.}, & \ldots, & q^{-\left(n-u_{k}-v_{k}-s\right)} \\
& q^{-(n-s)}, & q^{-\left(n-u_{1}-s\right)}, & \ldots, & q^{-\left(n-u_{k}-s\right)}
\end{array} ; q, q\right],
\end{aligned}
$$

where $a=n-\sum_{i=1}^{k} v_{i}$.

Proof. For each $i, 1 \leqslant i \leqslant k$, let

$$
f(i)=\left|\left\{j: u_{j} \leqslant i \leqslant u_{j}+v_{j}-1\right\}\right| .
$$

Define $M=\max \{m: f(m)>0\}$ and set $b=a+1-M-u_{1}$. Let $X$ be the subset of $\mathbb{N}$ defined by the binary sequence

$$
\tau=\underbrace{0 \ldots 0}_{b} \underbrace{1 \ldots 1}_{f(M)} 0 \underbrace{1 \ldots 1}_{f(M-1)} 0 \underbrace{1 \ldots 1}_{f(M-2)} 0 \ldots 0 \underbrace{1 \ldots 1}_{f\left(u_{1}+1\right)} 0 \underbrace{1 \ldots 1}_{f\left(u_{1}\right)} \underbrace{0 \ldots 0}_{u_{1}} .
$$

That is, let $i \in X$ if and only if $\tau_{i}=1$. The identity follows directly from Theorem 4.1 with $X$ as above and $Y=\mathbb{N}$.

Finally, we end this section by giving some examples showing that in some special cases, we can considerable simplify the formulas for the $P_{n, s}^{X, Y}(q)$.

Corollary 4.3. Let $X=2 \mathbb{N}$ and $Y=\mathbb{N}$. Then

$$
P_{2 n, s}^{X, Y}(q)=q^{s^{2}}\left([n]_{q} !\right)^{2}\left[\begin{array}{c}
n \\
s
\end{array}\right]_{q}^{2}
$$

which was originally derived by Liese and Remmel [5] using recursion (2.4). 
Proof. By the main theorem, we have

$$
\begin{aligned}
& P_{2 n, s}^{X, Y}(q)=[n]_{q} ! \sum_{r=0}^{s}(-1)^{s-r} q^{\left(\begin{array}{c}
s-r \\
2
\end{array}\right)}\left[\begin{array}{c}
n+r \\
r
\end{array}\right]_{q}\left[\begin{array}{c}
2 n+1 \\
s-r
\end{array}\right]_{q} \prod_{i=1}^{n}[r+i]_{q}
\end{aligned}
$$

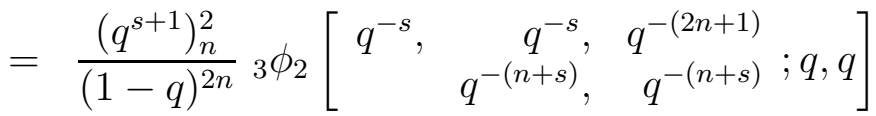

$$
\begin{aligned}
& =\frac{\left(q^{s+1}\right)_{n}^{2}}{(1-q)^{2 n}} \frac{\left(q^{n+1-s}\right)_{s}\left(q^{n+1-s}\right)_{s}}{\left(q^{n+1}\right)_{s}\left(q^{n+1}\right)_{s}} \\
& =q^{s^{2}}\left([n]_{q} !\right)^{2}\left[\begin{array}{c}
n \\
s
\end{array}\right]_{q}^{2},
\end{aligned}
$$

where we employ Jackson's $q$-analogue of the Pfaff-Saalschütz ${ }_{3} F_{2}$ summation formula (see [3])

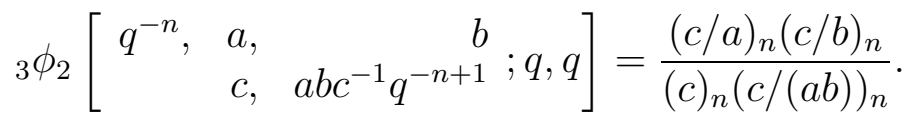

Remark 4.4. More generally, if $X=\{u+2, u+4, \cdots, u+2 m\}$ and $Y=\mathbb{N}$, a similar computation gives

$$
P_{2 m+u+v, s}^{X, Y}(q)=q^{s^{2}+v s}[m+u]_{q} ![m+v]_{q} !\left[\begin{array}{c}
m \\
s
\end{array}\right]_{q}\left[\begin{array}{c}
m+u+v \\
v+s
\end{array}\right]_{q} .
$$

Finally, we should note that it is possible to prove analogues of the results of this paper for the hyperoctahedral group $B_{n}$. We shall give such results in a subsequent paper.

\section{References}

[1] J. Liese, Counting Descents and Ascents Relative to Equivalence Classes mod $k$, Annals of Combinatorics 11 (2007) 481-506.

[2] G. Gasper, Summation Formulas for Basic Hypergeometric Series, Siam. J. Math. Anal., 12 (1981), 196-200.

[3] G. Gasper, M. Rahman, Basic Hypergeometric Series, in "Encyclopedia of Math. and its Applications," Cambridge Univ. Press, Cambridge, MA, 1990.

[4] J. Hall, J. Remmel, Counting Descents with Prescribed Tops and Bottoms, math.C0/0610608

[5] J. Liese, J. Remmel, $q$-Analogues of formulas for the number of ascents and descents with specified equivalences mod $k$, Permutation Patterns, 2006 